\title{
Application of the numerical method for the propagation of distributions to the calculation of coverage intervals in the thermovision measurements
}

by S. Dudzik*, W. Minkina

\begin{abstract}
* Faculty of Electrical Engineering, Institute of Electronics and Control Systems - Częstochowa University of
\end{abstract} Technology, Częstochowa, Poland

\section{Introduction}

In the contemporary measurement theory, the new methods of accuracy determination are still investigated. It is possible, that the theory of uncertainty is a universal tool for the estimation of accuracy $[1,2]$. This theory allows for the analysis of random interactions, even if the measurement model is strongly nonlinear. According to the theory of uncertainty, the standard uncertainty plays the main role in the accuracy determination.

The problem of determination of uncertainty components in thermovision measurement was described in [3-6]. In this paper the analysis of the measurement method accuracy, based on the coverage interval idea was presented. In the simulation research, the model described in $[4,5,7]$ was assumed. It can be formulated as a function of five input variables:

$$
T_{o b}=f\left(\varepsilon_{o b}, T_{a t m}, T_{0}, \omega, d\right),
$$

where: $\varepsilon_{o b}$ - object emissivity, $T_{a t m}$ - the temperature of atmosphere, $\mathrm{K}, T_{0}$ - ambient temperature, $\mathrm{K}$ $\omega$ - relative humidity, $d$ - distance between infrared camera and the object, $\mathrm{m}$.

In the research of components of combined standard uncertainty, by means model (1), Monte Carlo simulations were used. The expanded uncertainty, with the assumed level of confidence was calculated using the method for the propagation of distributions.

\section{The method for the propagation of distributions and Monte Carlo simulations}

The goal of the use the propagation of distributions is the determination of uncertainty, using Monte Carlo simulations. The fundamental purpose of computational procedure is to obtain a statistical coverage interval of the measurement model output variable. It is necessary to emphasize, that the results of calculations are correct, even if the model is strongly nonlinear and the probability density functions of output are asymmetric. This situation takes place in the thermovision measurements.

The Common Commitee for Basic Issues In Metrology took this into consideration and worked out "Supplement No.1" entitled "Numerical methods of propagation of distributions" [8]. "Supplement..." deals with evaluation of precision in indirect measurements, with particular emphasis on strongly nonlinear and/or complicated measurement models, like e.g. the processing algorithm of measurement path of an infrared camera. The method of propagation of distributions makes it possible to give a correct estimation of a measurement precision, in particular in the following cases [9]:

- $\quad$ the partial derivatives are unavailable,

- the distribution of the output variable is not Gaussian,

- the distributions of input variables exhibit assymetry,

- the measurement model is a strongly nonlinear function of input quantities,

- the uncertainty ranges of individual input quantities are incomparable.

The idea of the propagation of distributions is illustrated in Fig. 1. The symbols in Fig 1 denote: $g_{i}\left(\xi_{i}\right)-$ probability density functions of permissible values $\xi_{i}$ of the $i$-th input quantity $X_{i} ; g(\eta)$ - probability density function of permissible values $\eta$ of the measurement model output quantity $Y$.

The method for the propagation of distributions evaluates the uncertainties using Monte Carlo method. The principal aim of the computational procedure is to evaluate the statistical coverage interval at a specified confidence level. It is worth to emphasize that the procedure gives correct results even for strongly nonlinear functional relationships of measurement models as well as for assymetric probability dennsity functions of input random variables. The following steps can be distinguished in the evaluation of uncertainty: The method for the propagation of distributions consists of following steps [8]:

- Determination of the output random variable of the model.

- Determination of the input random variables of the model.

- Model design.

- Determination of the probability density function of the input variables.

- Calculation of the probability density function of the output variable using Monte Carlo simulations.

- Estimation of parameters of the probability density function of the output variable and $95 \%$ coverage interval.

1. The Monte Carlo method makes the numerical approximation of the cumulative distribution $G(\eta)$ of the output quantity possible. The simulation is based on the assumption that any value of an input quantity chosen at random from all permissible values of this input is as justified as any other. In other words, no value is preferred. Hence, drawing values of each input quantity according to the probability distribution function 
assigned to this input validates the set of its values. The value of the measurement model output corresponding to the drawn values of the inputs is a representative output. Consequently, a big enough set of the output values obtained from the model in this way can approximate, with required accuracy, the probability density distribution of permissible values of the output (the measured quantity). The Monte Carlo simulation is conducted in the following steps [8]:

2. Generation of a set of $N$ values by an independent sampling of the probabilisty density function of each input variable $X_{i}, i=1 \ldots N$. In the case of statistically dependent variables the samples must be generated with the use of the joint density function of these variables. The sampling is repeated $M$ times, where $M$ is a big number. As a result, we obtain $M$ independent sets of $N$ values of the inputs.

3. Simulation of the model for each set of values. As a result, we obtain a set of $M$ values (realizations) of the model output variable $Y$. This set is de facto a numerical approximation of the probability density distribution of the output variable.

4. Determination of the approximation $\hat{G}(\eta)$ of the cumulative density function $G(\eta)$ of $Y$, based on the generated set of values.

5. Evaluation of statistical parameters of the output variable distribution on the basis of $\hat{G}(\eta)$. In particular, the following are determined: the measured value $y$ as the expected value of $\hat{G}(\eta)$, the estimate of the standard uncertainty $u(y)$ - as the standard deviation of $\hat{G}(\eta)$, and the ending points of the confidence interval $I_{p}(y)$ for the assumed coverage probability - as two quantiles of $\hat{G}(\eta)$.

\section{The simulations of combined standard uncertainty in the thermovision measurements}

\section{Methodology of the simulation research}

The analysis of the model (1) should lead to the combined standard uncertainty estimation. The combined standard uncertainty characterizes unequivocally of the measurement accuracy in the statistical sense. In the paper, the method for the propagation of distributions, described above is used to estimate of the combined standard uncertainty. The simulation results of the object temperature uncertainty $u_{c}\left(T_{o b}\right)$ were obtained under assumption of the uniform probability distribution. The investigations were carried out for the exemplary uncertainties of the inputs. In the last stage of the accuracy analysis the $95 \%$ coverage interval of the output was estimated. This interval was calculated on the basis of the probability distribution obtained from simulations of the model (1) in accordance with the hints enclosed in [8].

The simulation research of the combined standard uncertainty was conducted 4 cases, depending on the $\varepsilon_{o b}$ and $T_{o b}$ estimates. It was assumed the two values of the object emissivity: 0,$4 ; 0,9$ and also two values of the object temperature: $323 \mathrm{~K}\left(50^{\circ} \mathrm{C}\right) ; 363 \mathrm{~K}\left(90^{\circ} \mathrm{C}\right)$. The results of the simulations are valid for the first measurement range for the typical infrared camera. For different camera measurement ranges the different calibration constants are valid. In the simulations, the model of the FLIR ThermaCAM PM 595 camera was used. In each case the 95\% coverage interval $I_{95 \%}$ was calculated. These intervals were compared with the coverage intervals on the assumption that the output variable has a normal distribution. The normal distribution is assumed for the output variable in most practical situations. In order to estimate the influence of the distribution asymmetry for the length of the $95 \%$ coverage interval, the dependence between this length and the order of the $\alpha$-quantile was presented. It enables the comparison of the minimal length of the $1_{95 \%}$ with the $1_{95 \%}$ under the assumption of the normal distribution of the output $(\alpha=0,025)$. Due to the determination of the $95 \%$ coverage interval it is necessary to set the value of the $\alpha$-quantile of the output variable. If the probability density function is symmetric, then the value of $\alpha$ can be expressed as:

$$
\alpha=\frac{1-p}{2},
$$

where: $p$ - the level of confidence (for example, $p=0,95(95 \%)$ corresponds to $\alpha=0,025)$.

\section{Results of the simulation research}

The simulation research of (1) was carried out using author's software created in the MATLAB environment. The data for simulations (the estimates of the input variables and their standard uncertainty) were collected in Table 1 and 2.

Table 1. Estimates of the input variables accepted in the combined uncertainty research of the model (1)

\begin{tabular}{|c|c|c|c|c|}
\hline $\begin{array}{c}\text { Object emissivity } \\
\left(\varepsilon_{o b}\right)\end{array}$ & $\begin{array}{c}\text { Ambient } \\
\text { temperature }\left(T_{0}\right)\end{array}$ & $\begin{array}{c}\text { Temperature of } \\
\text { atmosphere }\left(T_{a t m}\right)\end{array}$ & $\begin{array}{c}\text { Relative humidity } \\
(\omega)\end{array}$ & $\begin{array}{c}\text { Distance } \\
(d)\end{array}$ \\
\hline 0,$9 ; 0,4$ & $293 \mathrm{~K}$ & $293 \mathrm{~K}$ & 0,5 & $10 \mathrm{~m}$ \\
\hline
\end{tabular}


Table 2. Standard uncertainties of the input variables accepted in the combined uncertainty research of the model (1)

\begin{tabular}{|c|c|c|c|c|}
\hline $\begin{array}{l}\text { Object emissivity } \\
\left(\varepsilon_{o b}\right)\end{array}$ & $\begin{array}{c}\text { Ambient } \\
\text { temperature }\left(T_{0}\right)\end{array}$ & $\begin{array}{c}\text { Temperature of } \\
\text { atmosphere }\left(T_{\text {atm }}\right)\end{array}$ & $\begin{array}{c}\text { Relative humidity } \\
(\omega)\end{array}$ & $\begin{array}{c}\text { Distance } \\
(d)\end{array}$ \\
\hline 0,$09 ; 0,04(10 \%)$ & $9 \mathrm{k}(3 \%)$ & $9 \mathrm{~K}(3 \%)$ & $0,05(10 \%)$ & $1 \mathrm{~m}(10 \%)$ \\
\hline
\end{tabular}

Table 3. Coverage intervals calculated from Monte Carlo simulation and from the normal distribution

\begin{tabular}{|c|c|c|c|c|}
\hline $\mathcal{E}_{o b}$ & $T_{o b}, K$ & $u_{c}\left(T_{o b}\right)$ & I95\%-sym & l95\%-norm \\
\hline \multirow{2}{*}{0,9} & 323 & $2,9(0,9 \%)$ & {$[319,329] \mathrm{K}(10 \mathrm{~K})$} & {$[317,329] \mathrm{K}(12 \mathrm{~K})$} \\
\hline & 363 & $5,6(1,5 \%)$ & {$[355,373] \mathrm{K}(18 \mathrm{~K})$} & {$[352,375] \mathrm{K}(23 \mathrm{~K})$} \\
\hline \multirow{2}{*}{0,4} & 323 & $11(3,4 \%)$ & {$[302,341] \mathrm{K}(39 \mathrm{~K})$} & {$[300,344] \mathrm{K}(44 \mathrm{~K})$} \\
\hline & 363 & $9,9(2,7 \%)$ & {$[345,382] \mathrm{K}(37 \mathrm{~K})$} & {$[343,382] \mathrm{K}(39 \mathrm{~K})$} \\
\hline
\end{tabular}

In this paper the two $95 \%$ coverage intervals were compared. The first one was obtained from the distribution function of the output variable of the model (1). The second one was calculated considering, that the output variable has a normal distribution. The probability distributions of the output of the model (1), obtained from Monte Carlo simulations taking account above conditions are presented in Fig. 2, 4, 6, 8. The coverage intervals are collected in Table 3. The relationship between the $\alpha$ parameter and the $95 \%$ coverage interval was presented in Fig. 3, 5, 7, 9. The 95\% coverage interval obtained from Monte Carlo simulations was marked with solid line. The $95 \%$ coverage interval considering normal distribution was marked with broken line.

\section{Conclusions}

- The value of the combined standard uncertainty strongly increases with the decrease of the object emissivity $\varepsilon_{o b}$.

- Comparing the 95\% coverage intervals from the approximation of distribution function of output variable and from the assumption of normal distribution, one can observe that the differences are negligible.

- Indeed, as shown in Fig. $1-2$, the broken line of the coverage interval for the normal distribution is lying always under the solid line obtained from simulations.

- Taking into account of the above conclusion, it was found, that in the considered cases the assumption for the expanding factor $k$ equal to 2 is safe for the $95 \%$ confidence level.

- As can be seen from the presented simulation results, the asumption of $k=2$ leads to insignificant expansion of the $95 \%$ coverage interval in each case considered in the simulations.

- In other words, the confidence level of the interval determined under the assumption of the normal distribution of the output, is crossing $95 \%$. It confirms the thesis of the safety assumption of the normal distribution.

- The assumption of the normal distribution of the output variable of the model (1) - according to the central limit theorem - is safe from the viewpoint of the underflow of $95 \%$ coverage interval.

\section{REFERENCES}

[1] Taylor J. R.: "An Introduction to Error Analysis. The Study of Uncertainties in Physical Measurements", 2nd edition, University Science Books, Sausalito California, 1997.

[2] "Guide to the Expression of uncertainty in Measurement", ISO/TAG, 1995.

[3] Astarita T., Cardone G., Carlomagno G.M., Meola C.: "A survey on infrared thermography for convective heat transfer measurements", Optics \& Laser Technology, Vol. 32 (2000) pp. 593-610, Elsevier Science Ltd, 2000.

[4] Minkina W.: "Thermovision Measurements - Methods and Instruments", Technical University of Czestochowa Publishers, Czestochowa 2004, ISBN 83-7193-237-5 (in polish).

[5] Minkina W., Dudzik S.: "Simulation analysis of uncertainty of infrared camera measurement and processing path", Measurement - Vol. 39, Issue 8, pp 758-763, Elsevier Ltd, 2006.

[6] Dudzik S.: „The analysis of the influence of the correlation coefficient between the input variables on the uncertainty in the temperature determination by means infrared camera", proceedings of MKM'05 conference, Zielona Góra 5-7.09.2005r. pp. 195-203 (in polish).

[7] TOOLKIT IC2 Dig 16 „Developers Guide” 1.00 AGEMA 550/570. Reserved materials of FLIR company, made available for authors.

[8] "Guide to the Expression of Uncertainty in Measurement. Supplement 1: Numerical Methods for the Propagation of Distributions", Bureau International des Poids et Mesures (BIPM) 2004.

[9] Dudzik S., Minkina W.: „Estimation of the influence of input variables correlation in the thermovision measurement model with a low emissivity object", Measurements, Automatics and Control, 57 (2007), No. 2, pp. 29-32 (in polish). 


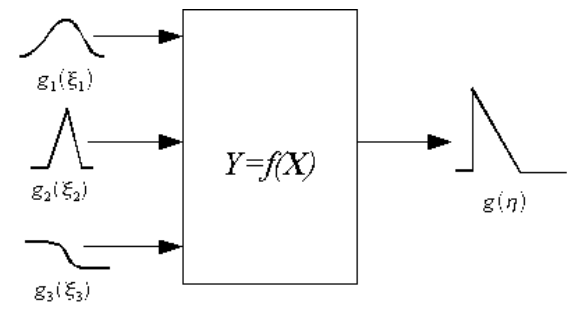

Fig. 1 Propagation of distributions [8]

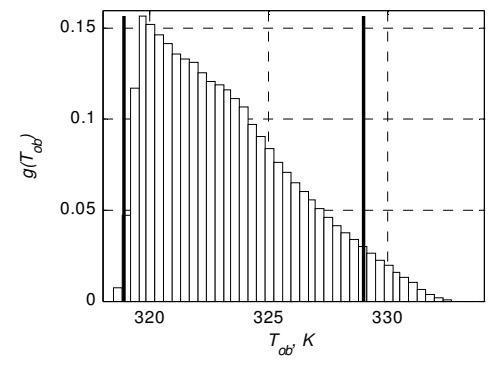

Fig. 2 The probability density function of the output variable in the model (1) for $T_{o b}=323 \mathrm{~K}$ and $\varepsilon_{o b}=0,9$

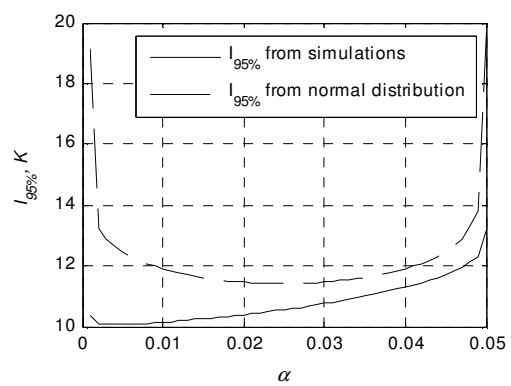

Fig. 3 The 95\% coverage interval as the function of $\alpha$ parameter for $T_{o b}=323 \mathrm{~K}$ and $\varepsilon_{o b}=0,9$

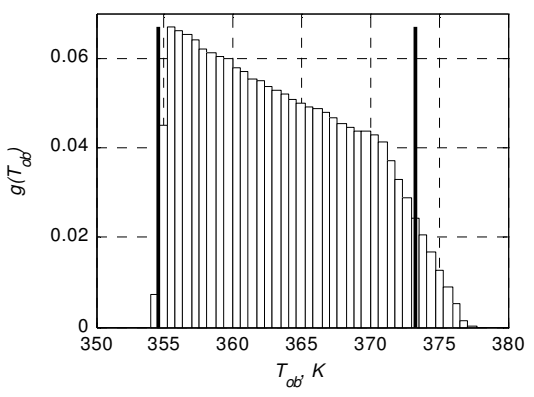

Fig. 4 The probability density function of the output variable in the model (1) for $T_{o b}=363 \mathrm{~K}$ and $\varepsilon_{o b}=0,9$

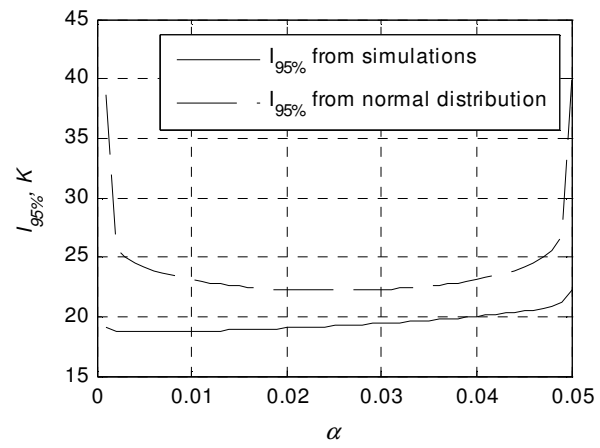

Fig. 5 The 95\% coverage interval as the function of $\alpha$ parameter for $T_{o b}=363 \mathrm{~K}$ and $\varepsilon_{o b}=0,9$ 


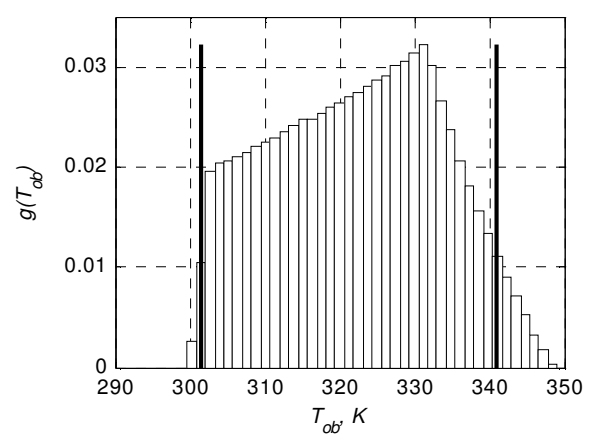

Fig. 6 The probability density function of the output variable in the model (1) for $T_{o b}=323 \mathrm{~K}$ and $\varepsilon_{o b}=0,4$

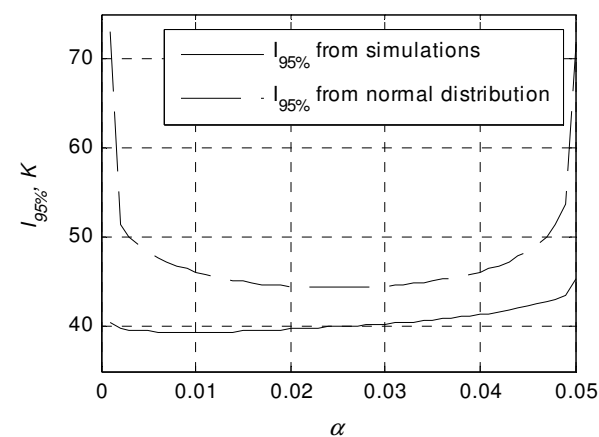

Fig. 7 The 95\% coverage interval as the function of $\alpha$ parameter for $T_{o b}=323 \mathrm{~K}$ and $\varepsilon_{o b}=0,4$

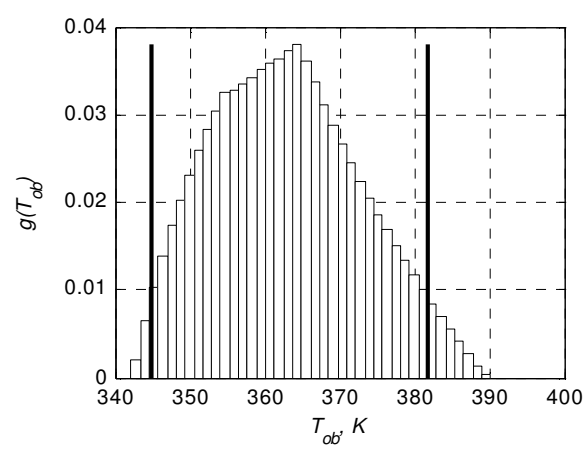

Fig. 8 The probability density function of the output variable in the model (1) for $T_{o b}=363 \mathrm{~K}$ and $\varepsilon_{o b}=0,4$

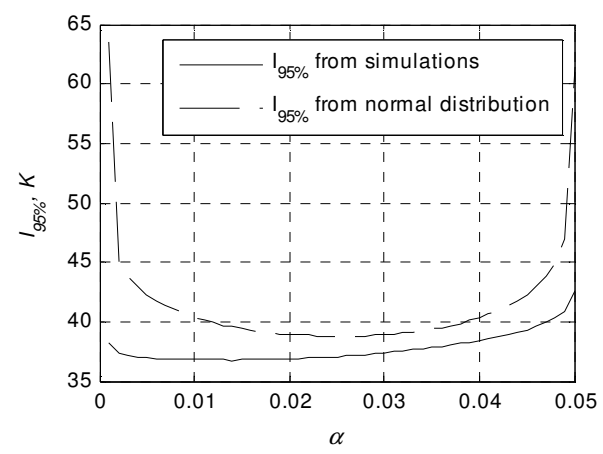

Fig. 9 The 95\% coverage interval as the function of $\alpha$ parameter for $T_{o b}=363 \mathrm{~K}$ and $\varepsilon_{o b}=0,4$ 
http://dx.doi.org/10.21611/qirt.2008.04_01_02 